\title{
Accelerated Electromigration Study of Cobalt Thin Films by In-Situ TEM
}

\author{
Brent Engler ${ }^{*}$, Robert Hull ${ }^{1}$ \\ 1. Rensselaer Polytechnic Institute, Materials Science and Engineering, Troy NY, USA \\ * Corresponding author: engleb2@rpi.edu
}

Cobalt has been studied in the interconnect field for years based on its utility as a capping layer for copper interconnects but only recently as the primary material in local interconnects, as dimensional scaling limits the effectiveness of traditional $\mathrm{Cu}$ interconnects $[1,2]$. Compared to $\mathrm{Cu}, \mathrm{Co}$ suffers from a higher bulk resistance but requires less liner material, has a higher resistance to stress voiding, a higher resistance to Electromigration (EM) failure, and better resistance scaling in low dimensions due to a longer electron mean free path [3]. The combination of the mean free path and decreased liner requirements result in a critical dimension where $\mathrm{Co}$ interconnects have a lower total resistance than $\mathrm{Cu}$ of the same dimension.

Cobalt has a complex EM behavior, and while it is generally known to be significantly more resistant to EM than $\mathrm{Cu}$, there is little data on the failure mechanisms at low dimensions. Because Co has a sign change in the normal Hall resistance, $\mathrm{R}_{0}$, above 400C [4], the direction of EM, indicated by the sign of the effective charge $Z^{*}$, also changes. The quantities $R_{0}$ and $Z^{*}$ do not change sign at the same temperature, as electron induced EM has been observed above 600C [5].

In-Situ TEM allows for unique insight into the mechanisms of EM with high temporal and spatial resolution and independent control over temperature and current density within a large range of operating conditions. In this work the conducting structures are fabricated over an electron transparent window of amorphous silicon nitride and proximal heat sink structures act to mitigate the effects of Joule heating. Many lines can be tested in parallel under nominally identical or varied thermal and geometrical parameters, and finite element modelling is used to calculate the temperature distribution over the sample geometry. The in-situ technique allows for real time observation of resistance change and mircostructural evolution to monitor EM in bulk and record the nucleation and growth/migration of voids.

Our EM test structures are fabricated as two interleaved Al combs with Co conducting lines connecting the two halves of the structure and the comb teeth serving to conduct heat away from the Co. A titanium nitride layer between the Co and the amorphous silicon nitride window acts as a diffusion barrier to the $\mathrm{Co}$ and an electrical shunt in the case of void formation. The test structures reside in an isolated ring furnace and are connected to electrical feed throughs in the holder by $\mathrm{Au}$ ball bonds made to the periphery of the Al comb structure. The electrical stimulus is controlled by a constant voltage DC power supply adjusted to maintain a constant current density through the $\mathrm{Co}$, and line temperature is controlled by the furnace setting and the Al-Co distance. Under stressing, constant temperature and current density through the sample, while both resistance changes and TEM observation provide evidence of EM. TEM imaging allows for tracking of voids in real time, Figure 1, and provides structural information to identify nucleation and growth/mobility behavior with respect to the Co microstructure.

In this work we demonstrate a technique for the measurement of in-situ Co electromigration under accelerated testing conditions and show observation of EM with a focus on the microstructural origins 
and morphological evolution of EM voids. Our experimental parameter space covers the range of temperature from $30 \mathrm{C}$ to $1000 \mathrm{C}$ and current density up to $1 \times 10^{7} \mathrm{~A} \mathrm{~cm}^{-2}$. This will contribute to a microstructural understanding and model of EM in low dimension Co structures across a wide range of temperature, current, and geometric conditions [6].

\section{References:}

[1] Van der Veen et al., 2018 IEEE International Interconnect Technology Conference (2018), p 172-4

[2] C.K. Hu et al., IEEE International Reliability Physics Symposium Proceedings (2018), p 4F.114F.16

[3] D. Gall, Journal of Applied Physics 119 (2016), p 085101 (5 pp.)

[4] G.J. Van Gurp, Journal of Physics and Chemistry of Solids 38 (1977), p 627-633

[5] G.J. Van Grup, Thin Solid Films 38 (1976), p 295-311

[6] This work was supported by the NYSTAR Focus Center at RPI, C150117, and made extensive use of cleanroom and characterization facilities in the Center for Materials, Devices and integrated Systems (cMDIS) at RPI.

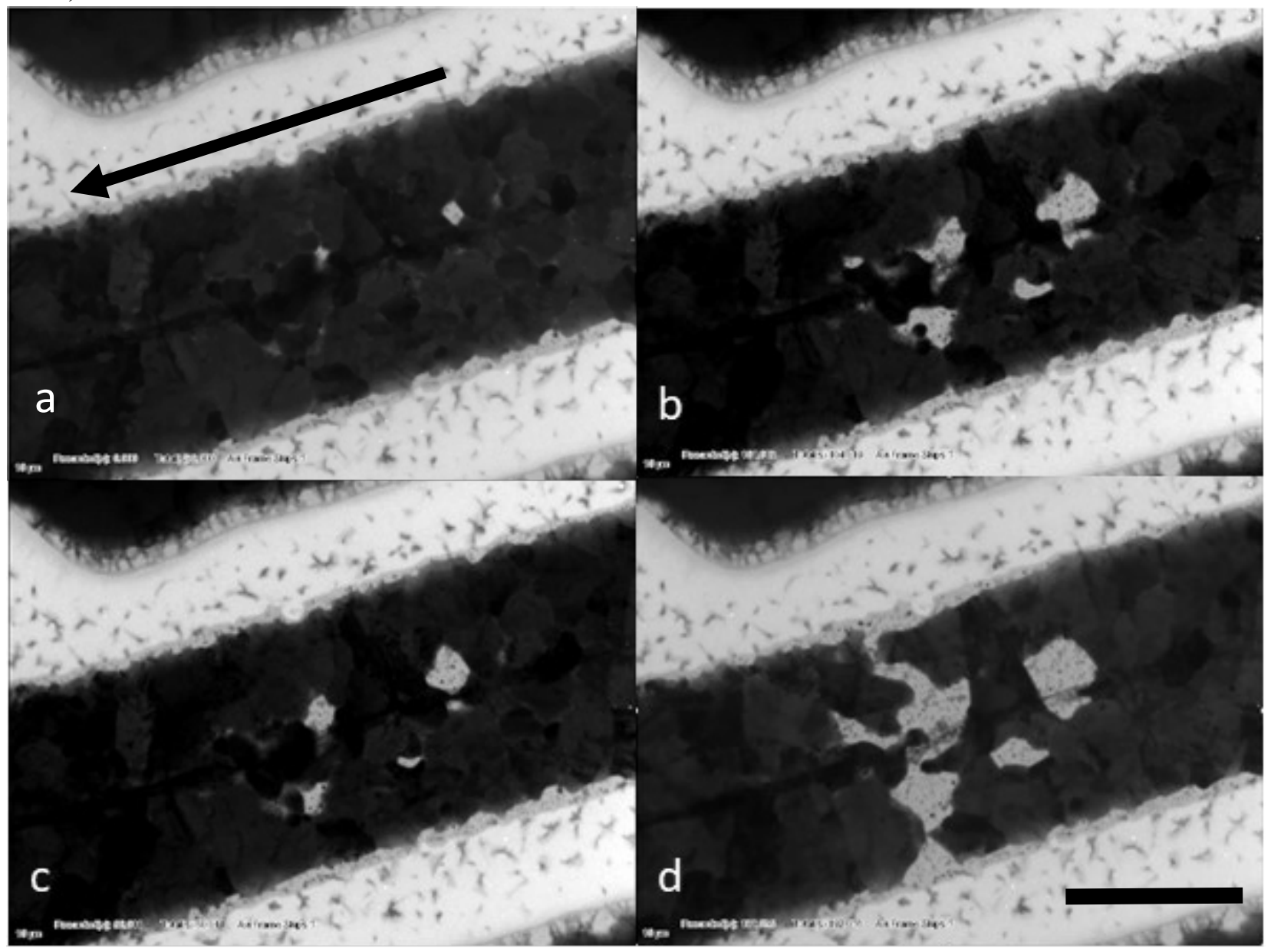

Figure 1. Time lapse of unpassivated Co line stressed at $5 \times 10^{6} \mathrm{~A} / \mathrm{cm}^{2}$ at an approximate temperature of $800 \mathrm{C}$ accounting for Joule heating. Electron flow is in the direction indicated in (a), and the scale bar is $2 \mu \mathrm{m}$. a) $\mathrm{t}=0 \mathrm{~s}, \mathrm{~b}) \mathrm{t}=60 \mathrm{~s}, \mathrm{c}) \mathrm{t}=104 \mathrm{~s}, \mathrm{~d}) \mathrm{t}=192 \mathrm{~s}$. Cathode directed electromigration resulting from $\mathrm{p}$-type conduction causes void nucleation and growth at the anode. 